PART IV

ABUNDANCE EFFECTS IN SPECTRAL CLASSIFICATION 


\title{
ABUNDANCE EFFECTS IN SPECTRAL CLASSIFICATION
}

\author{
C. JASCHEK \\ Observatoire de Strasbourg, France
}

\begin{abstract}
The groups discussed are:

O-type subdwarfs
CNO stars
He-strong stars
He-weak stars
B-type subdwarfs
Ap stars
Am stars
$\lambda$ Boo stars
$\delta$ Del stars
Fm stars
A-F-G stars with weak metal lines
Stars with CN anomalies
Stars with CH anomalies
Ba stars
\end{abstract}

Abstract. The paper attempts to put together the spectroscopic definitions used in the literature for different groups of peculiar objects linked with abundance anomalies.

In each case the useful range in dispersion is quoted and bibliographic references are provided.

When a system of spectral classification is set up, one automatically divides the stars into those that fit into the established scheme and those that do not. The latter ones are the peculiar objects with regard to the reference frame used. (Outside of this frame the designation has no meaning, because nothing is peculiar by itself). This situation lasts until a new classification scheme redefines the reference frame, re-shifting also the peculiarities according to the new physical insights.

Such a situation can be illustrated nicely with a group of peculiar stars - peculiar with regard to the HD system - called the ' $c$ ' stars. With the advances in the physics it turned out that these were luminous stars, and the next classification scheme, that of Morgan et al. (1943) incorporated all the former 'c' stars into a consistent scheme. This situation illustrates very well the need for using both theory and classification to advance in the field. Classification can tell only what happens and can set apart stars for further study, whereas theory should be able to tell the 'whys'.

I must admit that ihis picture is an oversimplification, because it omits the contributions of the photometrists and the astrometrists. If often happens that a group of peculiar objects is first defined by one characteristic and named by it, but that later on other characteristics are found which bear on most of the stars - but not on all - of the group. For instance, it turns out to be quite a task to know what is really meant when calling a star Am. It could be so-called because of discrepancies between the spectral types 
assigned to the $\mathrm{K}$-line, the Balmer lines and the metallic lines (i.e. because of its spectral classification); because of the ratio of two line-intensities (for instance $\mathrm{Sc} / \mathrm{Sr}$ ); because of the narrow band photometry performed, (for instance the Strömgren photometry); because of the multicolour photometry $(U B V R I)$ or because of an abundance analysis. A star called Am by one criterion is not necessarily so-called according to the others.

This is a very unfortunate situation because it gives a wrong point of departure for many investigations. In the particular case quoted, it is very difficult to single out Am stars photometrically, if there is no clear spectroscopic definition of what an Am star is.

Because of this situation, I will use my time to give a summarized version of the spectroscopic definitions of the various groups which are mentioned when talking of abundance efects. I have thought that such a documentation might be useful especially for the non-spectroscopists.

I said specifically 'spectroscopic definitions', because I think one can get a very clean description - and definition - just from the spectrum. I will thus try to avoid definitions implying theory; for instance I will not mention gravity or temperature. This is not only because I am not a theoretician, but because I think it is best to describe spectra such as they are observed. Take for instance luminosities of stars. Today everybody is used to roman numeral luminosity classes, without reference to the absolute magnitude related to that class. By taking 'luminosity' out of the hands of those working with distance scales, I think we have succeeded in concentrating more on what is seen in the spectrum. Because of the purely spectroscopic definitions, I will also exclude group assignments based on velocity, be it radial or space velocity. I wil therefore also not enter into the various population type assignments, except if they can be established from only an examination of the spectrum.

Therefore, in a certain sense, I am shrinking away from 'understanding' observations. This is true for all morphological science, be it botany, zoology, crystallography or spectral classification, and I accept voluntarily this self-imposed limitation. I think I can still speak about Am stars, despite ignoring if the effects are caused by abnormal abundances due to nuclear reactions, or to an abnormal atmospheric structure, or to diffusion processes, or to accretion, or to mass exchange between binary star components.

I mention this explicitly because since I am speaking about 'abundance effects in classification' I should know (but I don't) if all the groupss.I will be mentioning are really due to anomalous abundances. I think that in many cases this is likely, but we are not always sure, and it is for this reason that I shall include groups which perhaps some colleagues would not include if they had to talk about the subject.

I can be more definite about what I shall not include, for instance variable stars like Cepheids, RR Lyrae and novae. I will also omit binaries in which there are interactions between the components. I shall omit also stars which belong to the main groups of classification, like white dwarfs, carbon and $S$ stars and WR objects.

A question I would like to discuss before going into details is that of the line strengths. You will see that in many groups one speaks of a line - or lines - as being weaker than in a standard star (or in a 'normal star'). The degree of deviation from normalcy can be 
defined, like in the MK system, by means of a group of standard stars. This procedure has usually the difficulty that the list of standards is small, that the standards are not observable from the part of the sky and at the time of the year you are observing and that they do not cover the anomaly at all spectral types and luminosities. Another procedure is to refer the anomalies to another type of stars. One can say for instance that the spectrum is that of an A5 giant, but that the line at $\lambda 4215$ is enhanced as in an F5 III star. This procedure has the advantage that one can omit the selection of new standards for the Sr II line strength. Obviously the procedure fails if one tries to apply it to an exotic object like a mercury star, because the $\mathrm{Hg}$ line exists only in these stars, and not in the standards.

The last question I would like to mention is that each group is defined by what is seen at a given dispersion, so that it happens that at some dispersions the peculiarity becomes blurred and later on disappears when one uses different dispersions. I will thus quote what happens at each dispersion. The dispersion is given in $\AA \mathrm{mm}^{-1}$ at $\mathrm{H} \gamma$. I will add that there are some reasonable doubts if dispersion alone tells the whole story - if so, slit spectra and objective prism spectra of the same dispersion should give the same amount of information. However, this is only true if the spectra are also well enlarged, well exposed and if the seeing was good at the time the objective prism spectra were taken. I will not, however, go into more detail and assume simply that the dispersion by itself is the most important parameter.

Since Dr Seitter will describe in her paper what can be made out at dispersions between 240 and $1300 \AA \mathrm{mm}^{-1}$, I will restrict my description mostly to what can be seen at dispersion from 40 to $200 \AA \mathrm{mm}^{-1}$.

Finally I would like to point out that the bibliography indicated in each group is definitely incomplete. I have simply chosen those papers I found best suited to my purpose and I ask you to excuse any omission.

And now I will start with the descriptions.

\section{O-Type Subdwarfs (sdO)}

Balmer lines are broad when compared with dwarfs of similar spectral type; the number of Balmer lines is also smaller ( $n \sim 10-12$, compared to $n \sim 18$ in dwarfs). Furthermore, N IV ( $\lambda 3479$ for instance) is abnormally enhanced. If the ultraviolet spectrum is visible, group members can be detected at $180 \AA \mathrm{mm}^{-1}$ (Sargent and Searle, 1968). Greenstein and Eggen (1966) called attention to a possible division into line-rich and line-poor stars. Attention should be paid to the ratio $\lambda 4200: \lambda 4686$ of He II (Sargent and Searle, 1968) which might be smaller than in dwarfs. But on the whole He is normal or even stronger than in dwarfs (see also Graham and Slettebak, 1973). Another important element to examine, is S III.

These stars can be distinguished from B-type subdwarfs by the presence of $\lambda 4686$ 
He II. This line is generally not present in B-type subdwarfs. See, however, Baschek (1975) for two exceptions.

Several dozen of these stars are known.

\section{CNO Stars}

Under this name, we will group early type stars (O-B5) in whose spectra the lines of either $\mathrm{C}, \mathrm{N}$ and/or $\mathrm{O}$ are of abnormal intensity. The first stars of this group were described by Jaschek and Jaschek (1967); the group as such was defined by Walborn (1970) at 60 $\AA \mathrm{mm}^{-1}$. Although some stars can be detected even at $120 \AA \mathrm{mm}^{-1}$, higher dispersions should be used.

Since $\mathrm{C}, \mathrm{N}$ and $\mathrm{O}$ do have a positive luminosity effect (i.e. enhance in supergiants) CNO rich stars are easier to detect in the dwarfs and CNO deficient stars in supergiants.

It is possible that the group could be subdivided into 'carbon' and 'nitrogen' subgroups (Walborn, 1971).

A few dozen stars of this type are known.

\section{He Strong Stars}

(Also called simply 'He stars'). They are by definition stars in which $\mathrm{He}$ is very enhanced with respect to hydrogen, which is either weak or absent.

If the hydrogen is weak, but still exists, the He I spectrum shows singlet / triplet ratios similar to those in B-type dwarfs (for instance $\lambda 4387 / \lambda 4471$ ).

If the hydrogen is completely absent, no Balmer jump exists and the singlet / triplet ratios are different from those in dwarfs. Oxygen lines are usually absent in these objects and $\mathrm{C}$ is enhanced. This group is also called 'H-poor'.

The He-group was apparently first defined by Bidelman (1952). Further studies at high dispersion (by Hunger and co-workers, see Hunger 1975) have added many details, but no other outstanding common characteristics of the group.

About thirty stars of this group are known.

\section{He Weak Stars}

They can be defined as B-type stars in which the hydrogen-line spectrum and the helium spectrum disagree, the latter being weaker than in dwarfs of similar hydrogen-line spectrum. This definition is incomplete since many early Ap stars also do have weak helium lines. One should therefore add the condition '... and no strong Si II lines are visible'. The group was defined by Garrison (1967) on $90 \AA \mathrm{mm}^{-1}$ plates; Sargent and 
Searle (1968) at $50 \AA \mathrm{mm}^{-1}$ define a group of Bw stars, whose definition is practically equivalent to the present one, except that their definition is not purely spectroscopic. At the lower end one can distinguish stars of this type at $180 \AA \mathrm{mm}^{-1}$.

A subdivision of the group has been attempted by Jaschek and Jaschek (1974) and Baschek (1975) into:

(1) $\alpha \mathrm{Scl}$ type stars. Ti II and Fe II are visible together with a hydrogen line spectrum corresponding to about B5.

(2) P-stars. Stars in which P II lines are very enhanced.

(3) Blue helium stars. Si II is slightly stronger than normal.

Dispersions of at least $40 \AA \mathrm{mm}^{-1}$ are needed to distinguish members of these groups.

Attention is called to the possibility that many of the stars of this group are spectrum variables.

One important point here is that stars of this group can belong either to Population I or to Population II. In the latter case they are called horizontal branch stars. Greenstein and Sargent (1974) referring to the latter, state that 'either one or more of He I, C II and $\mathrm{Mg}$ II are weakened; generally $\mathrm{He} \mathrm{I}$ is weak', and that the rotational velocity is low. Sargent and Searle (1968) stress that He I weakness does not correlate with the behaviour or the lines of heavier elements.

At the present is does not seem possible to distinguish in a purely spectroscopic way whether a star belongs to the Population I or II. In particular the Balmer line spectrum does not give any clue. Incidentally this group provides a good example of the interaction between definitions and theory. Horizontal branch stars in the solar neighbourhood are picked out as such because of their He-weakness. But it is argued sometimes that all horizontal branch stars are helium weak, which might be true but cannot be ascertained from material selected in the way described.

About $10^{2}$ objects of this type are known.

\section{B-Type Subdwarfs (sdB)}

In this group, Balmer lines are too broad for the spectral type and the number of Balmer lines is smaller than in dwarfs ( $n \sim 10-12$, as compared with $n \sim 18$ for normal dwarfs). Often, but not always, the He I lines are weak.

The group was defined by Greenstein and Münch around 1954, but I have been unable to trace.the exact origin. Members of this group can be detected on $180 \AA \mathrm{mm}^{-1}$ plates (or lower), if the Balmer jump is visible. When observed at $50 \AA \mathrm{mm}^{-1}$, Sargent and Searle (1968) call attention to anomalies in the singlet/triplet ratio of the helium lines. So, for instance, $\lambda 4387 / \lambda 4471$ is smaller than in dwarfs.

Greenstein and Sargent (1974) studied a sample of these stars at higher dispersion (around $20 \AA \mathrm{mm}^{-1}$ ). Several anomalous objects were found, but no regularity emerges. Perhaps the $\lambda 4253$ S III is important for drawing subgroups.

About $10^{2}$ objects are known. 


\section{Ap Stars}

They were described as a group for the first time by Morgan (1933) (using $30 \AA \mathrm{mm}^{-1}$ spectrograms) as being late B- or early A-type stars in whose spectrum some lines are very enhanced. On the basis of the elements whose lines are most intensified, Morgan introduced six main groups, namely the $\mathrm{Mn}, \lambda 4200, \mathrm{Cr}$, Eu, Sr and Si stars. This sequence is given in order of advancing spectral type, except for the Si-stars, which are scattered through the whole group. In recent years one more group, that of the $\mathrm{Hg}$ stars, has been introduced (Bidelman) and the sequence has been slightly rearranged. Also recent authors tend to list all elements whose lines are enhanced, not only the most enhanced one.

The following groups can be regarded as being the classical ones. They are listed in order of advancing spectral type and some of the most characteristic lines of each element are provided.

$\begin{array}{ll}\mathrm{Si}-\lambda 4200 & \lambda 4200,3955 \\ \mathrm{Mn}(\mathrm{II}) & \lambda 4137,4206 \text { also } \lambda \lambda 3441,3460,3474 \\ \mathrm{Hg}(\mathrm{II}) & \lambda 3984 \\ \mathrm{Si}(\mathrm{II}) & \lambda 4128-31,3854,56-62 \\ \mathrm{Cr}(\mathrm{II}) & \lambda 4171, \text { blend around } 4111,4233 \\ \mathrm{Eu}(\mathrm{II}) & \lambda 4129,4205,3930 \\ \mathrm{Sr}(\mathrm{II}) & \lambda 4077,4215\end{array}$

Not all of these families can be detected efficiently at all dispersions. Although extreme cases of $\mathrm{Si}, \mathrm{Cr}$ and $\mathrm{Sr}$ stars can be detected at $300 \AA \mathrm{mm}^{-1}$ (Honeycutt and McCuskey, 1966), the groups become reasonably well defined only at $120 \AA \mathrm{mm}^{-1}$ (Cowley et al., 1969) except for the $\mathrm{Mn}$ and $\mathrm{Hg}$ stars which are still difficult. Dispersions of at least $60 \AA \mathrm{mm}^{-1}$ (Osawa, 1965) should be used to overcome this. The Mn-group is easier to detect in the ultraviolet region, through the lines of $M .3(\lambda 3441,3460,3474)$ (Nariai, 1967).

In the last decades the simple classification scheme outlined has become more complicated because at higher dispersion more intermediate groups were added. So Osawa (1965) uses 19 groups, most of them being combinations of the seven listed above.

With regard to other characteristics of the Ap stars, the hydrogen-line spectrum seems to be comparable to that of dwarfs of similar type, both in the line shape and the Balmer jump. If helium lines are visible, they tend to be weak for the type assigned to the hydrogen lines (Deutsch, 1956).

Attention must be called to the large percentage of spectrum variables in this group, which makes impossible the assignment of a unique classification of each object, unless non-variability is proven.

Attempts have been made to simplify the subdivisions within this group (see for 
instance Jaschek and Jaschek, 1974). Usually the subdivision is made into Si stars, Mn stars and Eu-Cr-Sr stars (also called rare earth objects).

It should be added that it is in the latter group where most of the outstanding composition anomalies are found, such as the presence of very heavy elements, up to uranium (Jaschek and Malaroda, 1970), and the elements characteristic of nuclear fission (see review by Kuchowicz, 1973).

The number of known Ap stars is large $\left(n>1 \times 10^{3}\right)$.

\section{Am Stars}

They were discovered by Titus and Morgan (1940) in the Hyades and several of them were described in the Atlas (Morgan et al., (1943). At $100 \AA \mathrm{mm}^{-1}$ one can classify them according to the $\mathrm{Ca}$ II $\mathrm{K}$ line, to the hydrogen lines and to the metallic lines. If one calls these types respectively $S(K), S(H)$ and $S(m)$, one has $S(K) \leqslant S(H) \leqslant S(m)$.

Furthermore the metallic lines are sharp and some lines usually strengthened in giants ( $\lambda 4077 \mathrm{Sr}$ II, Fe II $\lambda 4173=\lambda 4178$ ) are also enhanced in the Am's. This pseudo-luminosity effect resulted in these objects being classified as giants. However there are differences from the spectrum of true giants, especially $\lambda 4417 \ll \lambda 4481$. (Cowley et al., 1969).

This description applies to dispersions between 100 and $40 \AA \mathrm{mm}^{-1}$ but at higher dispersions care must be taken to compare with stars of low rotational velocity.

Stars with marginal Am characteristics are often called 'mild Am'.

At $20 \AA \mathrm{mm}^{-1}$ higher, the ratio $\lambda 4246 \mathrm{Sc} \mathrm{II} / \lambda 4215 \mathrm{Sr}$ II has been used (Bidelman, 1956; Conti, 1965) to characterize Am stars. It must, however, be stressed that not all Sc II weak stars are Am stars (in many Ap stars of the Cr-Eu-Sr type, Sc II weak) and that not all Am stars are Sc II weak.

At lower dispersions, one can pick out extreme Am stars (with $S(K) \ll S(m)$ ) up to $300 \AA \mathrm{mm}^{-1}$ (Honeycutt and McCuskey, 1966).

Many Am stars are known $\left(n>10^{3}\right)$.

\section{8. $\lambda$ Boo}

The star $\lambda$ Boo, prototype of the group, was first described by Morgan et al. (1943) in the Atlas, at $110 \AA \mathrm{mm}^{-1}$. For the Balmer line strengths, all other metallic lines are abnormally weak, including those of Ca II.

At higher dispersions it becomes evident that the lines are not sharp and that the velocity is small; Slettebak et al. (1968) used this as a discriminant from horizontal branch stars. For a description of the group at higher dispersion see Baschek and Searle (1969).

Less than ten objects are assigned to this group. 


\section{9. $\delta$ Sct}

The first object of this group was described by Roman (1951), but most of the stars were discovered photometrically later on. Their spectra seem about normal for early F-type giants except for the weakness of the Ca II lines. (Bidelman, 1951). Morgan and Abt (1972), who have studied spectroscopically for the first time a large sample of $\delta$ Sct Stars, state that these stars have ".. Luminosity classes brighter than class $V$ and that the spectra of these $\delta$ Sct stars do not form a homogeneous group. (The Ca II lines show a great range in intensity among objects having similar spectral types and luminosity classes)'. These stars can be detected between $40 \AA \mathrm{mm}^{-1}$ and $120 \AA \mathrm{mm}^{-1}$.

A few dozen stars of this type are known.

\section{Fm Stars}

Bidelman discovered the first star of this type, studied by Preston (1961). Later on a few more stars were discovered at $80-100 \AA \mathrm{mm}^{-1}$, characterized by a metallic-line spectrum which corresponds to a later type than that indicated by the hydrogen lines. The $G$ band corresponds to an even more advanced type than the metallic lines. Generally $\mathrm{Sr}$ II is strong and Ca II slightly earlier than the hydrogen lines.

The denomination Fm introduced by Houk (1975) points to the relation with Am stars. There are reasonable doubts if this is really an independent group.

A few dozen stars of this type are known.

\section{A, F and G Dwarfs with Weak Metal Lines}

Morgan et al. (1943) mentioned for the first time stars in which the metallic lines are weaker than normal. Later on, Roman (1954) described the existence of a group of weak-lined $F$ stars. She added that the behaviour of the hydrogen spectrum, including the number of visible lines and the jump, were all compatible. She studied then a sample of bright F5-G5 stars in which she found the so-called 'weak-line stars' defined at $125 \AA \mathrm{mm}^{-1}$ (Roman, 1952) as stars having weak metals, but no specific definition was provided. Morgan (1958) called attention to the fact that the procedure is extremely sensitive to misclassification. If for instance an F7 star is called F8, it might be called later on 'metal weak'.

Greenstein (1960) states

the characteristic feature of the spectra of these stars is the extreme weakness of metallic lines for the temperature given by the star's colour and by the level of excitation and ionization. The hydrogen lines are strong and have relatively sharp and deep cores. Turbulence must be small from the few data given so far by curves of growth and from the visual appearance of extreme line sharpness. Rotation is small or absent.

Although this is not a purely spectroscopic description, it indicates that the essential 
feature is the discrepancy between the spectral type corresponding to metallic lines and to the hydrogen lines.

It should be added incidentally that some, but not all, RR Lyrae stars are metal weak.

Bond (1970) made a survey of metal deficient, i.e. weak metallic line, stars. He selected them from a sample of stars found on objective prism plates, at $110 \AA \mathrm{mm}^{-1}$. He found that the easiest objects to find are those of type $F$ and $G$; at spectral type $A$ excellent seeing is needed, to prevent the faint lines from disappearing. He analyzed then some objects on slit spectrograms, with dispersions ranging between 77 and $142 \AA \mathrm{mm}^{-1}$. The criteria he used are

... to classify the stars primarily on the basis of their hydrogen line strengths. In some cases the strength and appearance of the $G$ band were also taken into account.... Luminosity classes were assigned primarily on the basis of the ratio of Sr II $\lambda 4077$ to the nearby Fe I lines and, in the earlier stars, by the ratio of the blend near $\lambda 4172$ (mostly Fe II) to Ca I $\lambda$ 4226. ...Once a star had been classified, its spectrum was again compared with the standard of the same type (if available) and the degree of weakening of the metallic lines (principally in the range $\lambda \lambda 3933-4226$ ) was estimated. Four adjectives were used - none, slight, moderate, extreme.

With lower dispersions, extreme cases can still be found at $200 \AA \mathrm{mm}^{-1}$ according to Graham and Slettebak (1973), like the K2 star mentioned in the publication.

On the other hand, Wilson (1961) used a dispersion of about $10 \AA \mathrm{mm}^{-1}$ to analyse possible difference in late G- and early K-type dwarfs. He found two characteristics namely that some stars do possess emissions in the core of the $\mathrm{Ca}$ II lines and second, that for equal intensity of the hydrogen lines, the strength of the metallic lines can be very different. Stars exhibiting these phenomena are probably related to the more extreme cases found with lower dispersion at earlier spectral types.

Several hundreds of stars of this type are known.

\section{CN Anomalies}

Although CN had been used as a luminosity criterion by Lindblad back in 1922, it was Morgan and Keenan who pointed out in the Atlas (1943) that both CN and metals were weak in some high-velocity stars. The $\mathrm{CN}$ effects are noticeable in giants of type G7-K2, because it is in this region where the $\mathrm{CN}$ bands are normally strong enough to become conspicuous features of the spectrum, whereas $\mathrm{CN}$ is never that conspicuous in dwarfs.

The $\mathrm{CN}$ strong stars were discovered by Morgan and Nassau on objective prism plates; they .were later called $\lambda 4150$ stars by Roman (1952).

Since usually the $\lambda 4216 \mathrm{CN}$ band is more easily visible than both the $\lambda 4150$ and/or the $\lambda 3889$, Keenan and Keller (1953) used at $100 \AA \mathrm{mm}^{-1}$ the ratio $\lambda 4216 / \lambda 4172$. $\lambda 4216$ is a blend of $\lambda 4215 \mathrm{Sr}$ II and $\lambda 4215 \mathrm{CN}$, whereas $\lambda 4172$ is a blend of metallic lines. When $\lambda 4216$ is weak (i.e. CN weak), then the index is small. Keenan (1961 and 1964) recommended then the introduction of the ' $\mathrm{CN}$ discrepancy' $=\mathrm{CN}_{*-} \overline{\mathrm{CN}}$ which is sometimes also called the ' $\mathrm{CN}$ anomaly'. He thinks that this index is a consistent indicator of metal abundance in stars later than G5. (Morgan and Keenan, 1973). A list of stars 
with various degrees of $\mathrm{CN}$ discrepancy (between +3 and -3 ) defines the observational status. One should observe that Keenan defines the strength of $\lambda 4215$ on the basis of the intensity ratios:

$$
\begin{aligned}
& I(4211-13): I(4219-21), \text { whereas Schmitt (see below) uses } \\
& I(4210-16): I(4215-27)
\end{aligned}
$$

What photometrists measure when dealing with $\mathrm{CN}$ is something different and it is well illustrated in Golay (1975).

Schmitt (1971) used the following definition of a 'CN strong' star - '... is a star whose spectrum shows more absorption in the $0-1$ seq. of the $\mathrm{CN}$ band with its head at $\lambda 4216$ than the average MK standard shows at the same spectral type and luminosity class'. His stars were selected from a list of $\lambda 4150$ stars found on objective prism plates at $120 \AA \mathrm{mm}^{-1}$ and observed later on slit spectrograms $\left(\sim 100 \AA \mathrm{mm}^{-1}\right)$.

Later on, $\mathrm{CN}$ strong stars were called metal rich and $\mathrm{CN}$ weak stars either population II stars, or metal poor stars. While this might be right, it is also true that it is best to adhere strictly to what is observed (CN anomalies) rather than to generalize. This is also important in view of the fact that there exists at least one instance in which a dwarfs has weak metallic lines and strong CN. (Wilson, 1961, at $10 \AA \mathrm{mm}^{-1}$ ).

It should be added finally that $\mathrm{CN}$ strong stars tend to have strong $\mathrm{Ca} I \lambda 4226$, a strong $\mathrm{G}$ band and sometimes weak $\mathrm{H} \gamma$.

With regard to dispersions, a range of $80-150 \AA \mathrm{mm}^{-1}$ can be used to detect these stars. A large number of objects is known $\left(n>10^{3}\right.$ stars).

\section{CH Anomalies}

We will group here all stars in which the $\mathrm{CH}$ bands have an anomalous intensity. Usually it is the $G$ band which is most conspicuous and usually these stars are giants, of the types G-K.

The first object of this group was found by Keenan and Morgan (1941) at $120 \AA \mathrm{mm}^{-1}$; the group as such was described by Keenan (1942) who called them $\mathrm{CH}$ stars. These stars are characterized by very strong bands due to $\mathrm{CH}$, including an extremely strong $G$ band, and considerably weaker lines of neutral metals than in typical 'carbon' stars. The features are so strong as to suppress even the $\lambda 4226 \mathrm{Ca} I$ line. Usually, but not always, $C_{2}$ and $\mathrm{CN}$ are strong in these objects. $\lambda 4077$ and 4215 of Sr II are always strong and usually $\lambda 4554 \mathrm{BaII}$ is present. These stars are closely related to the carbon stars.

Later on N. Houk (1975) found at $110 \AA \mathrm{mm}^{-1}$ two or three dozens of such stars, although the enhancement is less intense than in the previous group.

On the other hand there exists also a group of stars in which $\mathrm{CH}$ is very weak or absent. This is accompanied usually by very weak hydrogen lines and strong $\mathrm{C}_{2}$ and $\mathrm{CN}$ 
bands and strong CI lines. The class was first described by Bidelman (1953) at $80 \AA \mathrm{mm}^{-1}$. These stars are also called 'hydrogen-poor carbon stars'.

Recently Bond (1974) added a new subgroup, namely that of the subgiant $\mathrm{CH}$ stars. He describes them as $\mathrm{G}$ type spectra with weak metallic lines but enhanced features of $\mathrm{CH}$ and strong Sr II $\lambda 4077$ and 4215 lines. In some extreme cases, the metallic lines are so weak that the $\mathrm{Sr}$ II lines, the $\mathrm{CH}$ features, the Balmer lines and sometimes $\lambda 4554$ are the only features clearly seen longward of the $\mathrm{Ca}$ II $\mathrm{K}$ and $\mathrm{H}$ lines.

Stars of this type are also detectable at $110 \AA \mathrm{mm}^{-1}$ (Houk, 1975). Less than a hundred ' $\mathrm{CH}$ anomalous' objects are known.

\section{Ba Stars}

A K-type giant with a strong $\lambda 4554 \mathrm{Ba}$ II line is called a barium star. The group was first described by Bidelman and Keenan (1951) at $80 \AA \mathrm{mm}^{-1}$ although isolated examples were known before. These authors add that all the Ba II stars show, in addition '... a definite enhancement of the $\mathrm{G}$ band and also, probably, of the violet bands of $\mathrm{CN}$ as well. The lines of Sr II at $\lambda 4077$ and $\lambda 4215$ (the latter blended with the head of the $(0.1) \mathrm{CN}$ band) are also strengthened'. Also the $C_{2}$ band $\lambda 5165$ is stronger than in normal stars.

At higher dispersion most of the elements heavier than strontium are overabundant. Warner (1965) at $80 \AA / \mathrm{mm}$ introduced a notation of Ba II enhancement, with steps from 1 to 5 , the latter one being the most extreme enhancement.

McConnell et al. (1972) at a dispersion of $110 \AA \mathrm{mm}^{-1}$ used the criterion of the 'prominence of the $\mathrm{Ba}$ II line at $\lambda 4554$, the strength of $\lambda 4077$ relative to $\mathrm{H} \delta$, and the strengths of the $\mathrm{CN}$ band ( $\lambda$ 4215) and the G band.'

They use no 'Ba strength' notation, but use the terms 'certain' and 'marginal' Ba II stars.

They also call attention to a few stars which are probably borderline cases between $C$ and $\mathrm{Ba}$ II stars. Another subgroup might be one of 'weak lines in their Fe group elements, when compared with MK standards and other Ba stars'.

Several hundreds of objects are known.

\section{Acknowledgements}

Finally I would like to thank my colleagues W. P. Bidelman, N. Houk and Mercedes Jaschek for their friendly criticism during the preparation of this paper.

\section{References}

Baschek, B.: 1975, in Problems in Stellar Atmospheres and Envelopes, Springer-Verlag. Baschek, B. and Searle, W. L. W.: 1969, Astrophys. J. 155, 537. 
Bidelman, W. P. and Kecnan, P. C.: 1951, Astrophys. J. 114, 473.

Bidelman, W. P.: 1951, Astrophys. J. 113, 304.

Bidelman, W. P.: 1952, Astrophys. J. 116, 227.

Bidelman, W. P.: 1953, Astrophys. J. 117, 25.

Bidelman, W. P.: 1956, private communication.

Bond, H. E.: 1970, Astrophys. J. Suppl. 22, 117.

Bond, H. E.: 1974, Astrophys. J. 194, 95.

Conti, P. S.: 1965, Astrophys. J. 142, 1594.

Cowley, A., Cowley, C., Jaschek, M. and Jaschek, C.: 1969, Astron. J. 74, 375.

Deutsch, A.: 1956, Publ. Astron. Soc. Pacific 68, 92.

Garrison, R. F.: 1967, Astrophys. J. 147, 1003.

Golay, M.: 1974, in Introduction to Astronomical Photometry, D. Rcidel Publ. Co., p. 254.

Graham, J. A. and Slettebak, A.: 1973, Astron J. 78, 295.

Greenstein, J. L.: 1960, in Stellar Atmospheres, Univ. of Chicago Press, p. 707.

Grcenstein, J. L. and Eggen, O.: 1966, Vistas Astron. 8, 63.

Greenstcin, J. L. and Sargent, A. I.: 1974, Astrophys. J. Suppl. 28, 157.

Houk, N.: 1976, this volume, p. 127.

Hunger, K.: 1975, in Problems in Stellar Atmospheres and Envelopes, Springer-Verlag.

Honeycutt, R. K. and McCuskey, S. W.: 1966, Publ. Astron. Soc. Pacific 78, 289.

Jaschek, M. and Jaschek, C.: 1967, Astrophys. J. 150, 355.

Jaschek, M. and Malaroda, S.: 1970, Nature 225, 246.

Jaschck, M. and Jaschek, C.: 1974, Vistas Astron. 16, 131.

Keenan, P. C.: 1971, I.A.U. Trans 9, 404.

Keenan, P. C.: 1964, I.A.U. Trans 10, 447.

Keenan, P. C. and Morgan, W. W.: 1941, Astrophys. J. 94, 501.

Kuchowicz, B.: 1973, Quart. J. Roy. Astron. Soc. 14, 121.

MacConnell, D. J., Frye, R. L., and Upgren, A. R.: 1972, Astron. J. 77, 384.

Morgan, W. W.: 1933, Astrophys. J. 77, 330.

Morgan, W. W., Keenan, P. C., and Kellman, E.: 1943. An Atlas of Stellar Spectra, Univ. of Chicago Press.

Morgan, W. W.: 1958, in D. J. K. O’Connell S. J. (eds), Stellar Populations, Vatican Observatory, p. 263.

Morgan, W. W. and Abt, H. A.: 1972, Astron. J. 77, 35.

Morgan, W. W. and Keenan, P. C.: 1973, Ann. Rev. Astron. Astrophys. 11. 29, Palo Alto.

Nariai, K.: 1967, Publ. Astron. Soc. Japan 19, 180.

Osawa, K.: 1965, Ann. Tokyo Astron. Obs. (2) 9, 123.

Preston, G. W.: 1961, Astrophys. J. 134, 797.

Roman, N.: 1951, Astrophys. J. 113, 705.

Roman, N.: 1952, Astrophys. J. 116, 122.

Roman, N.: 1954, Astron. J. 59, 307.

Sargent, W. L. W. and Searle, L.: 1968, Astrophys. J. 152, 443.

Schmitt, J. L.: 1971, Astrophys. J. 163, 75.

Slettebak, A., Wright, R. R., and Graham, J. A.: 1968, Astron. J. 73, 152.

Titus, J. and Morgan, W. W.: 1940, Astrophys. J. 92, 257.

Walborn, N.: 1970, Astrophys. J. 161, L149.

Walborn, N.: 1971, Astrophys. J. 164, L67.

Warner, B.: 1965, Monthly Notices Roy. Astron. Soc. 129, 263.

Wilson, O. C.: 1961, Astrophys. J. 133, 457.

\section{DISCUSSION}

Bidelman: Bond's supposed luminosity class IV $\mathrm{CH}$ stars do indeed appear to be nuclearly-evolved 
stars of quite low luminosity. They can also be considered hotter and less luminous Ba II stars. They raise very important questions.

With regard to the so-called rare-earth Ap stars, it should be emphasized that there are appreciable differences between the species of rare earths that are overabundant in the $\mathrm{Sr}$-Eu stars like $\gamma$ Equulei and the $\mathrm{Cr}$-Eu stars like $\beta$ Coronae Borealis. This difference should not be forgotten.

The Ba II stars published by MacConnell and Upgren, which I had some hand in, probably include quite a few stars similar to Bond's low-luminosity $\mathrm{CH}$ stars. Eggen has done photometry of some of the new Ba II stars and has concluded from this that many may not really be Ba stars. This conclusion is probably not entirely warranted in view of the above.

Williams: With reference to Dr Bidelman's comment on Eggen's photometry of the Ba II stars, he may like to know that I have carried out a spectroscopic analysis of one Ba II star not supported as such by Eggen's photometry and confirmed its overabundance of barium and other heavy metals. I do not think broad band photometry is a good discriminant of Ba II stars.

Walborn: I would like to comment on the complexity of the phenomena within the He-strong group. First, among the 'intermediate' group (which I prefer to call 'helium-rich' to avoid an implication of continuity with the extreme group), there appears to be a continuous range of helium enhancement, from slightly stronger than in nurmal stars, to almost as strong as the hydrogen lines. Secondly, within the extreme group there is a large range of gravities and also of CNO-line behaviour. The gravities appear to range from intermediate between those of subdwarfs and normal mainsequence stars, to as low as those of giants. While I think most extreme helium stars have C-enhanced, there are also examples of both $\mathrm{C}$ and $\mathrm{N}$ strong (HD 160641) and of $\mathrm{N}$ enhanced (BD $+13^{\circ} 3224$ ). So there are probably objects with a variety of evolutionary histories in this category.

Hack: Could you say what is the rotational velocity of He-weak stars on the average?

Jaschek: Low. Usually below $100 \mathrm{~km} \mathrm{~s}^{-1}$.

Cayrel de Strobel: You said that you do not link population criteria, but if you put a 'sd' or VI on an F star, you do.

Walborn: The problem with luminosity class VI is that it has not been systematically defined. However, if it is used, it should mean that there is some luminosity criterion in the spectrum which indicates a luminosity lower than class $\mathrm{V}$.

Morgan: (to Dr. Cayrel): Can you distinguish subdwarfs from dwarfs exclusively from spectroscopic means?

Foy: First, we can obtain the effective temperature using $T_{\text {eff }}$ of a given star by hydrogen line profiles $\mathrm{H} \alpha$ or $\mathrm{H} \beta$ (if the star is not too cool).

Second, we obtained a relation between $T_{\text {eff }}$ and the gravity $g$ by imposing ionization equilibrium, i.c. we determine $T_{\text {eff }}$ and $\log g$ so that for a given element, the abundance derived from neutral lines agrees with that observed from ionized lines.

Third, we obtained another independent relation between $T_{\text {eff }}$ and $\log g$ : it comes by imposing to find the same abundance for a given element either from weak lines of the curve of growth or from wing profiles of strong lines of this element. Magnesium is a good candidate to this criterion, because wings of $\mathrm{Mg}$ Ib triplet are very sensitive to gravity.

The intersection of these three criteria gives us $T_{\text {eff }}, \log g$ and $[\mathrm{M} / \mathrm{H}]$ : the value of $\log g$ indicates to us whether the star is a subdwarf $(\log g>4.80$ at solar spectral type $)$ or not.

Baschek: I would like to comment on a problem concerning the spectroscopic distinction of sdO from sdB stars. The strength of He II 4686 depends on the temperature as well as on the helium abundance. There are some stars ('OB-type subdwarfs' HD 149382, Feige 66) which exhibit weak (neutral) He which is characteristic of $\mathrm{sdB}$, but also He II 4686 which is characteristic of sdO.

Jaschek: Thanks for your observation. This is another case of objects intermediate between two groups. 